NOTES

\title{
A Study on the Reactivity of Carboxypropyl Group on Telechelic Poly(dimethylsiloxane)
}

\author{
Shigeru NoBE and Yasuyuki TezuKA* \\ Department of Material Science and Technology, Nagaoka University of Technology, \\ Kamitomioka, Nagaoka, Niigata 940-21, Japan
}

(Received February 18, 1994)

\begin{abstract}
KEY WORDS Telechelic Poly(dimethylsiloxane) / Carboxypropyl End Group / Back-Biting Mechanism / Esterification / Caesium Carboxylate / Alkaline Treatment /
\end{abstract}

Uniform-size polymers possessing reactive end groups are of a significant importance as macromolecular building blocks for the synthesis of precisely designed block and graft copolymers. In this context, we have synthesized a series of uniform-size poly(DMS)s, having various reactive end groups for the preparation of well-defined multi-component polymer materials containing poly(DMS) segment. ${ }^{1}$

We have recently developed a new initiator system, ${ }^{2}$ by which a bifunctional living poly(dimethylsiloxane), poly(DMS), can be produced through anionic ring-opening polymerization of hexamethylcyclotrisiloxane $\left(D_{3}\right)$. The use of a dilithium salt of bis ( $p$-dimethylhydroxysilyl)phenyl ether as an initiator and the addition of an appropriate amount of $\mathrm{N}$-methylpyrrolidine is the key of the living polymerization to occur. The subsequent endcapping reaction of living poly(DMS) with (3-dimethylchlorosilyl)butanoic acid trimethylsilyl ester could provide a bifunctional, uniform-size poly(DMS) having carboxylic acid end groups (2). ${ }^{3}$

Since carboxylic acid end groups can be readily converted to carboxylate salt group by the treatment of alkaline reagent, telechelic
poly(DMS) having carboxylate salt end groups will be produced. It can be utilized subsequently for various polymer reactions, including macromolecular ion-coupling reactions ${ }^{4}$ by the enhancement of nucleophilic reactivity of carboxylate anion in organic medium as in the phase transfer catalyst systems. ${ }^{5,6}$ On the other hand, siloxane bond in poly(DMS) is known to be susceptible to be cleaved by the action of acid or alkali catalysts. $^{7,8}$ Thus in the present study, we examined the reactivity of a series of alkali metal carboxylate end groups introduced on telechelic poly(DMS).

\section{EXPERIMENTAL}

\section{Materials}

Poly(DMS) having carboxylic acid end groups was prepared by the method detailed before. ${ }^{2,3}$ Molecular weights of the poly(DMS) samples used in the present study were 4900 (sample No. 1), 5500 (sample No. 2), and 4500 (sample No. 3), respectively.

Alkaline Treatment of Poly(DMS) Having
Carboxypropyl End Groups (2)
Into a $300 \mathrm{ml}$ separatory funnel, $50 \mathrm{ml}$ of $1 \mathrm{~N}$

* To whom correspondence should be addressed. 
$\mathrm{NaOH}$ and $1.5 \mathrm{~g}$ of 2 (sample No. 1) dissolved in $15 \mathrm{ml}$ of ethyl ether were mixed. The mixture was shaken for $30 \mathrm{~min}$ at ambient temperature, and allowed to stand for $24 \mathrm{~h}$ to separate the organic layer from aqueous phase. The aqueous layer was extracted three times with ethyl ether. The organic layer was collected and washed with saturated $\mathrm{NaCl}$ solution. After ethyl ether was removed by evacuation, the reaction product was isolated by precipitation into methanol, and finally freeze-dried from benzene solution. The yield was $1.4 \mathrm{~g}(93 \%)$. The acid treatment of 2 (sample No. 3, $0.5 \mathrm{~g}$ ) was carried out by a similar manner with $50 \mathrm{ml}$ of $1 \mathrm{~N} \mathrm{HCl}$. The yield was $0.43 \mathrm{~g}(86 \%)$.

\section{Reaction of Poly(DMS) Having Carboxypropyl \\ End Groups (2) after Alkaline Treatment}

In a $30 \mathrm{ml}$ round-bottom flask, $0.63 \mathrm{~g}$ of 2 (sample No. 2) was dissolved in $10 \mathrm{ml}$ of tetrahydrofaran (THF). Thereupon an equimolar amount of aqueous $\mathrm{NaOH}(0.1 \mathrm{ml})$ was added and stirred for $5 \mathrm{~h}$ at ambient temperature. Then a 3-fold excess of chlorodimethylvinylsilane and triethylamine were added to perform the end-capping reaction with stirring for $1 \mathrm{~h}$ at ambient temperature. The reaction mixture was first filtered with a G-5 glass filter and condensed by the evacuation, followed by the precipitation into methanol containing $0.1 \mathrm{~N}$
$\mathrm{HCl}$ (vol. ratio 9/1). The recovered product was purified by precipitation into methanol, and finally freeze-dried from benzene solution. The yield was $0.34 \mathrm{~g}(54 \%)$. The end-capping reaction with methyl iodide was performed for $24 \mathrm{~h}$ at ambient temperature after the treatment with an equimolar amount of a series of aqueous alkaline solution $(0.1 \mathrm{ml})$.

\section{Measurements}

$270 \mathrm{MHz}{ }^{1} \mathrm{H}$ NMR spectra were recorded by means of a JEOL JNM-GX270 apparatus at $40^{\circ} \mathrm{C}$ in $\mathrm{CDCl}_{3}$. Chemical shift values were calibrated from the solvent signal of $\mathrm{CDCl}_{3}$ $\left(\delta=7.30 \mathrm{ppm}\right.$ for $\left.{ }^{1} \mathrm{H}\right)$. GPC measurements were carried out using a TOSOH Model CCPD High Liquid Chromatograph equipped with RI8000 and UV8000 (254 $\mathrm{nm})$ as detectors, and with a TSK G3000HXL as a column. THF was used as eluent. IR spectra were taken on a Shimadzu FT-IR 8100 apparatus.

\section{RESULTS AND DISCUSSION}

Carboxylic acid end groups of poly(DMS) were converted to carboxylate salt end groups by the treatment of an excess amount of a series of alkaline reagents (Scheme 1). Telechelic poly(DMS)s having carboxylate salt end groups with enhanced nucleophilic reactivity

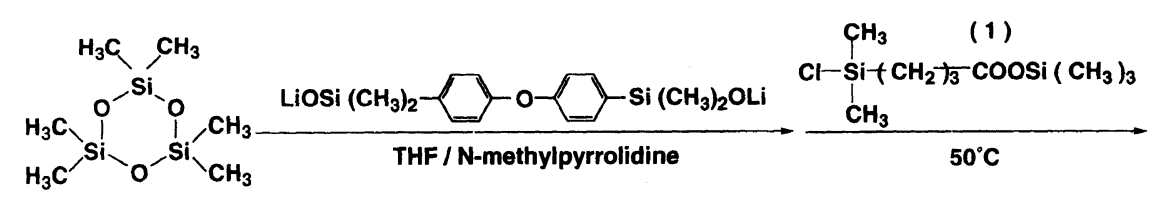<smiles>C[Si](C)(CCC(=O)O)O[Si](C)(C)O[Si](C)(C)c1ccc(Oc2ccc([Si](C)(C)[Si](C)(C)O[Si](C)(C)O[Si](C)(C)CCC(=O)O)cc2)cc1</smiles>

(2)

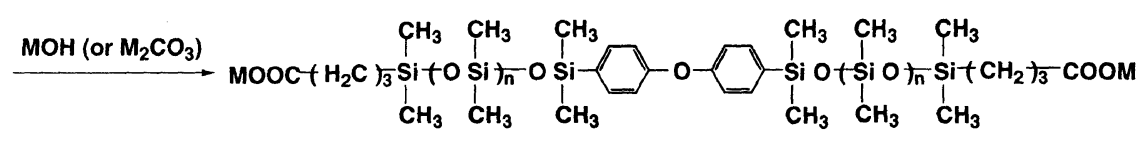

M: Li, Na, K, Rb, Cs

Scheme 1. 


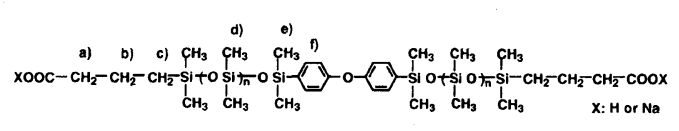

(c)

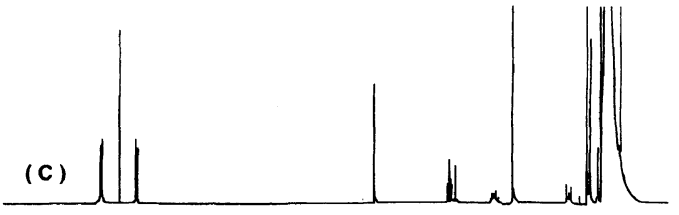

(B)
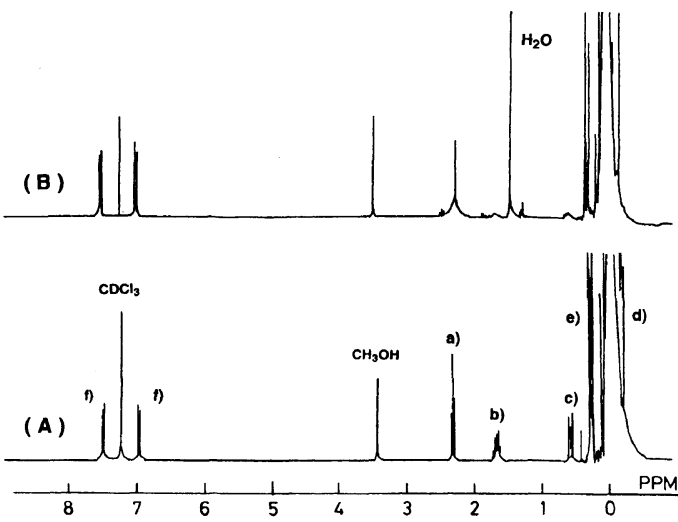

Figure 1. $\quad 270 \mathrm{MHz}^{1} \mathrm{H}$ NMR spectra of (A) poly(DMS) having carboxylic acid end groups, 2, (B) the product after the treatment with $1 \mathrm{~N} \mathrm{NaOH}$ and (C) the product after the further treatment with $1 \mathrm{~N} \mathrm{HCl}$. (2: sample No. 1, in $\left.\mathrm{CDCl}_{3}, 40^{\circ} \mathrm{C}\right)$.

are expected to be utilized for various polymer reactions, including macromolecular ioncoupling reaction. ${ }^{4}$

In the IR spectra of the product, the absorption at $1720 \mathrm{~cm}^{-1}$ visible in the starting 2, due to free carboxylic acid groups disappeared by the alkaline treatment and insteadly that at around $1600 \mathrm{~cm}^{-1}$ appeared with overlapping of phenyl group absorption. This is certainly indicative of the formation of carboxylate salt end groups. GPC inspection also showed that any noticeable broadening due to the random scission of siloxane linkage in poly(DMS) is absent in the product.

However, an elimination reaction of carboxypropyl end groups was indicated by ${ }^{1} \mathrm{H}$ NMR analysis of the product. In Figure 1, the spectrum of the original 2 having carboxylic acid end group and that of the product after the alkaline treatment are compared. Since
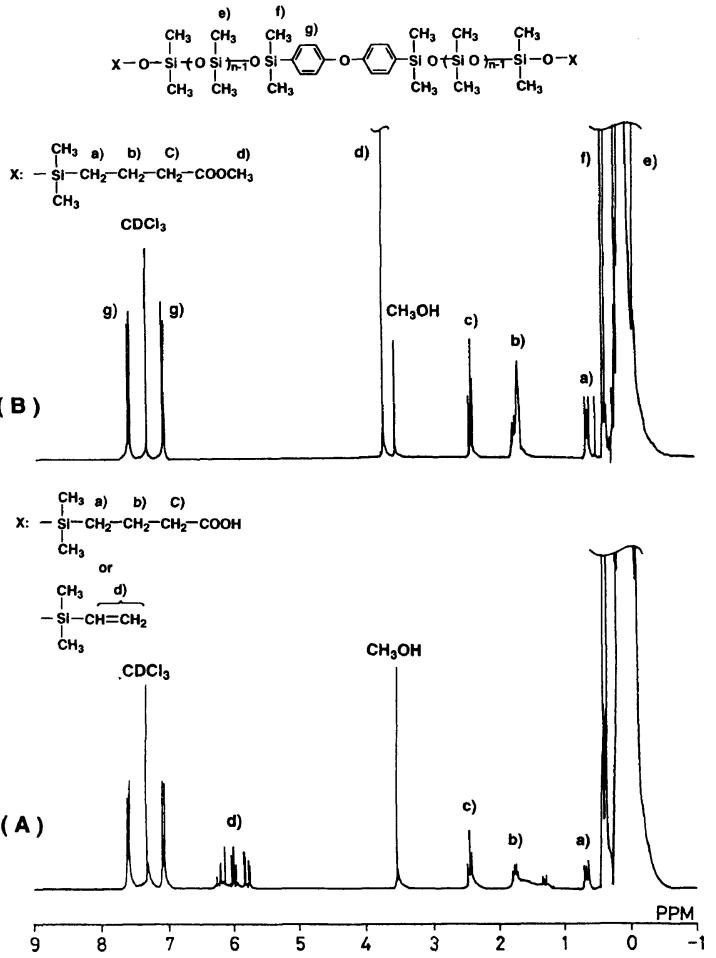

Figure 2. $270 \mathrm{MHz}^{1} \mathrm{H} \mathrm{NMR}$ spectra of (A) the reaction product after the treatment of 2 with $\mathrm{NaOH}$, followed by the treatment with chlorodimethylvinylsilane, and (B) the reaction product after the treatment of 2 with $\mathrm{CsOH}$, followed by the treatment with methyl iodide (A: sample No. 2, B: Run 5 in Table I, in $\mathrm{CDCl}_{3}, 40^{\circ} \mathrm{C}$ ).

carboxypropyl signals in carboxylate salt groups suffered notable broadening presumably due to the aggregation of polar ionic end groups, the relative content of carboxylate salt end groups with reference to the initiator fragment was estimated after the further treatment with $1 \mathrm{~N} \mathrm{HCl}$ solution to regenerate carboxylic acid groups. Although 2 itself was confirmed to be stable by $1 \mathrm{~N} \mathrm{HCl}$ treatment, notable decrease of the content of carboxypropyl end groups was observed by comparing the signal intensity of the end groups with that of the initiator fragment, i.e., diphenyl ether group at $7-8 \mathrm{ppm}$. This is indicative of an elimination reaction of end group to occur during the alkaline treatment.

The further details on the elimination 
reaction of the carboxypropyl end groups in poly(DMS), were then studied. Thus, 2 was treated first with an equimolar amount of $\mathrm{NaOH}$, and subsequently with chlorodimethylvinylsilane in the presence of triethylamine. ${ }^{1} \mathrm{H}$ NMR spectrum of the recovered product is shown in Figure 2(A). Signals due to vinyl silane groups were observed at $5.8-6.3 \mathrm{ppm}$, which were introduced through the reaction of silanol (or silanolate) end groups with chlorodimethylvinylsilane. Signals due to the residual carboxypropyl groups were also observed. By taking into account also for the GPC result, that the peak profile of the product remained unaffected during the alkaline treatment, the present elimination reaction is considered to take place through a back-biting mechanism via a conformationally favored 6-menbered ring transition state which may be formed specifically with carboxypropyl end groups, as shown in Scheme 2.

To deal with the adversarial elimination reaction of carboxylate salt end groups on poly(DMS), we then studied esterification reaction with methyl iodide by using various types of alkaline reagents, namely a series of alkali( $\mathrm{Li}, \mathrm{Na}, \mathrm{K}, \mathrm{Rb}, \mathrm{Cs})$ hydroxides or carbonates. The results are summarized in Table I. Although the elimination reaction was observed in most cases, the type of alkali metal employed in the reaction was found to influence significantly on the selectivity in the formation of the ester group. With caesium reagents, in particular, methyl ester end groups were produced in high selectivity. The ${ }^{1} \mathrm{H}$ NMR spectrum of the reaction product in Figure 2(B) shows a singlet signal at $3.70 \mathrm{ppm}$ due to methyl protons of methyl carboxylate end groups, and the comparison of the signal intensity with that of the initiator fragment indicates almost quantitative esterification reaction to occur with caesium hydroxide. IR analysis also confirmed the esterification reaction to occur. Thus the absorption at $1720 \mathrm{~cm}^{-1}$ due to carboxylic acid groups disappeared and that at $1750 \mathrm{~cm}^{-1}$ due to

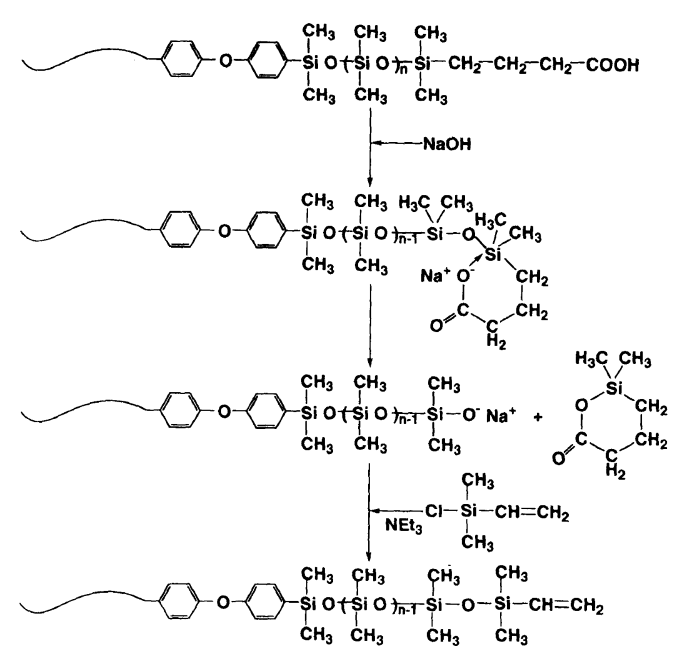

Scheme 2.

Table I. Reaction of poly(DMS) having carboxylate salt end groups with methyl iodide ${ }^{a}$

\begin{tabular}{rlcc}
\hline Run & $\begin{array}{c}\text { Alkaline } \\
\text { reagent }\end{array}$ & $\begin{array}{c}\text { Recovered } \\
\text { yield } / \%\end{array}$ & $\begin{array}{c}\text { Degree of } \\
\text { esterification } / \%^{\mathbf{b}}\end{array}$ \\
\hline 1 & $\mathrm{LiOH}$ & 76 & 89 \\
2 & $\mathrm{NaOH}$ & 69 & 41 \\
3 & $\mathrm{KOH}$ & 70 & 40 \\
4 & $\mathrm{RbOH}$ & 75 & 92 \\
5 & $\mathrm{CsOH}$ & 81 & 100 \\
6 & $\mathrm{Li}_{2} \mathrm{CO}_{3}$ & 45 & 0 \\
7 & $\mathrm{Na}_{2} \mathrm{CO}_{3}$ & 65 & 10 \\
8 & $\mathrm{~K}_{2} \mathrm{CO}_{3}$ & 78 & 67 \\
9 & $\mathrm{Rb}_{2} \mathrm{CO}_{3}$ & 75 & 63 \\
10 & $\mathrm{Cs}_{2} \mathrm{CO}_{3}$ & 80 & 90
\end{tabular}

a 2 (sample No. 2); $0.5 \mathrm{~g}, \mathrm{THF} ; 10 \mathrm{ml}, \mathrm{H}_{2} \mathrm{O} ; 1 \mathrm{ml}$, methyl iodide; $0.08 \mathrm{~g}$. For the detailed reaction procedure, See EXPERIMENTAL.

${ }^{b}$ By ${ }^{1} \mathrm{H}$ NMR.

methyl ester groups was formed along with the reaction. In addition, no peak broadening was observed in GPC analysis.

In conclusion, telechelic poly(DMS) having caesium carboxylate end groups was found to be useful prepolymer, which underwent a nucleophilic substitution reaction with methyl iodide to produce ester end groups. Thus this poly(DMS) prepolymer will be applicable to further reactions to produce functional polymers containing polysiloxane segment. 
Acknowledgments. We thank Toshiba Silicone Co. for the gift of silicon compounds. A financial support from Izumi Science and Technology Foundation is gratefully acknowledged.

\section{REFERENCES}

1. Y. Tezuka, Progr. Polym. Sci., 17, 471 (1992).

2. H. Kazama, Y. Tezuka, and K. Imai, Polym. Bull., 21, 31 (1989).
3. H. Kazama, Y. Tezuka, and K. Imai, Macromolecules, 24, 122 (1991).

4. Y. Tezuka, T. Shida, T. Shiomi, K. Imai, and E. J. Goethals, Macromolecules, 26, 575 (1993).

5. Y. Tezuka and E. J. Goethals, Makromol. Chem., 188, 783 (1987).

6. W. P. Weber and G. W. Gokel, in "Phase Transfer Catalysis in Organic Chemistry," Springer-Verlag, Heiderberg, Germary, 1977.

7. N. Mougin, P. Rempp, and Y. Gnanou, Makromol. Chem., 194, 2533 (1993).

8. J. Chojnowski and M. Mazurek, Makromol. Chem., 176, 2999 (1975). 